\title{
Pauli potential in terms of kinetic energy density and electron density in the leading Coulombic term of the nonrelativistic $1 / Z$ expansion of spherical atomic ions
}

\author{
N. H. March ${ }^{1,2}$ and Á. Nagy ${ }^{3}$ \\ ${ }^{1}$ Department of Physics, University of Antwerp, Antwerp, Belgium \\ ${ }^{2}$ Oxford University, Oxford, England \\ ${ }^{3}$ Department of Theoretical Physics, University of Debrecen, H-4010 Debrecen, Hungary
}

(Received 7 October 2009; published 25 January 2010)

\begin{abstract}
The Pauli potential $V_{P}$ in density functional theory is known to be the difference between the functional derivative of the single-particle kinetic energy $T_{s}[n]$ with respect to the electron density $n$ and its von Weizsäcker counterpart. For the leading Coulombic term in the $1 / Z$ expansion for spherical atomic ions, $V_{P}[n]$ is written in terms of the kinetic energy density plus $n(r)$ and its low-order derivatives. For comparison, the example of an arbitrary number of closed shells with purely harmonic confinement is also treated.
\end{abstract}

DOI: 10.1103/PhysRevA.81.014502

PACS number(s): 31.15.E-

In a recent article in this journal [1] the differential virial theorem has been written in single-particle density functional theory (DFT) in terms of the first derivative of the Pauli potential $V_{P}$ for spherical atomic densities $n(r)$. This allows the so-called force-balance equation to be written in the form

$$
\begin{aligned}
-n(r) \frac{\partial V}{\partial r}= & -\frac{\hbar^{2}}{4 m} \frac{\partial}{\partial r} \nabla^{2} n(r)+4 \frac{t_{W}(r)}{r} \\
& +2 \frac{\partial t_{W}(r)}{\partial r}+n(r) \frac{\partial V_{P}}{\partial r}
\end{aligned}
$$

where $t_{W}(r)$ is the von Weizsäcker kinetic energy density: $\frac{\hbar^{2}}{8 m}(\nabla n)^{2} / n$.

Here, to make further analytical progress in understanding the Pauli potential, we shall obtain $V_{p}^{\prime}(r)$ from Eq. (1) for the leading Coulombic term in the important $1 / Z$ expansion [2] for spherical atomic ions. In other words, we are considering a bare Coulomb field approximation, where the generalized form of Kato's theorem is valid. In this Coulomb problem, with $V(r)$ in Eq. (1) becoming $V(r)=-Z e^{2} / r$, one of us [3] has derived the spatial generalization of Kato's theorem [4] as

$$
\frac{\partial n(r)}{\partial r}=-\frac{2 Z}{a_{0}} n_{0}(r),
$$

where $n_{0}(r)$ is the $s$-wave $(l=0)$ component of the total density $n(r)$ for an arbitrary number of closed shells. Inserting Eq. (2) in Eq. (1) for the Coulomb potential, we readily obtain

$$
\begin{aligned}
& n(r) V_{P}^{\prime}(r)+4 \frac{t_{W}(r)}{r}+2 \frac{\partial t_{W}(r)}{\partial r} \\
& =-n(r) \frac{Z e^{2}}{r^{2}}-\frac{\hbar^{2}}{2 m} \frac{Z}{a_{0}} \frac{\partial}{\partial r}\left[n_{0}^{\prime}(r)+\frac{2}{r} n_{0}(r)\right] .
\end{aligned}
$$

If we denote the kinetic energy density of this problem by $t_{G}(r)$ where $G$ denotes the wave function form $(\nabla \psi)^{2}$, we next employ the result of Amovilli and March [5], readily obtaining

$$
\frac{\partial t_{G}(r)}{\partial r}=\frac{\hbar^{2}}{8 m} n^{\prime \prime \prime}(r)-\frac{3 \hbar^{2}}{4 m r^{2}} n^{\prime}(r)-\frac{3 Z e^{2}}{2 r^{2}} n(r) .
$$

Using Eq. (4) in Eq. (3) to remove the term $-n(r) \frac{Z e^{2}}{r^{2}}$, it follows that

$$
\begin{aligned}
& n(r) V_{P}^{\prime}(r)+4 \frac{t_{W}(r)}{r}+2 \frac{\partial t_{W}(r)}{\partial r} \\
& =\frac{2}{3} \frac{\partial t_{G}(r)}{\partial r}-\frac{1}{3} n_{0}^{\prime \prime}(r) \frac{Z}{a_{0}} \frac{\hbar^{2}}{m}-\frac{Z}{a_{0} r} \frac{\hbar^{2}}{m} n_{0}^{\prime}(r) .
\end{aligned}
$$

While it is convenient, especially for a large number of closed shells, to work with the $s$-state density $n_{0}(r)$, in fact use again of Eq. (2) gives back the more compact DFT-like formula that

$$
\begin{aligned}
& n(r) V_{P}^{\prime}(r)+4 \frac{t_{W}(r)}{r}+2 \frac{\partial t_{W}(r)}{\partial r} \\
& =\frac{2}{3} \frac{\partial t_{G}(r)}{\partial r}+\frac{\hbar^{2}}{6 m} n^{\prime \prime \prime}(r)+\frac{\hbar^{2}}{2 m} \frac{n^{\prime \prime}(r)}{r} .
\end{aligned}
$$

Since it is known from the theorems of DFT [6] that $t_{G}$ is a functional of the density $n(r)$, it is to be emphasized that Eq. (6) gives, at least in principle, $V_{P}^{\prime}(r)$ as a functional of the electron density $n$. This is, of course, a central objective of DFT for the Pauli potential.

Heilmann and Lieb [7] have shown what to us is the remarkable fact that if we sum specifically the squares of the hydrogenic wave functions over the entire bound-state level spectrum we obtain a density, say, $n_{\infty}$, which is everywhere finite. Of course, it is so long range that it is naturally not normalizable, the density $n_{\infty}$ as $r \rightarrow \infty$ giving the semiclassical Thomas-Fermi result

$$
n_{\infty}=\kappa r^{-3 / 2} \quad r \rightarrow \infty,
$$

where $\kappa=2^{1 / 2}\left(3 \pi^{2}\right)^{-1}$ [8]. [Also $n_{\infty}(0)=\frac{1}{\pi} \sum_{n=1}^{\infty} n^{-3} \approx$ 0.383.] Inserting this into Eq. (4) we find in this asymptotic limit

$$
\frac{\partial t_{G \infty}}{\partial r}=-33 \frac{\hbar^{2}}{64 m} \frac{\kappa}{r^{9 / 2}}-\frac{3 e^{2}}{2} \frac{\kappa}{r^{7 / 2}} \quad r \rightarrow \infty .
$$

Combining Eqs. (1) and (7) we arrive at the result

$$
V_{P \infty}^{\prime}=-\frac{e^{2}}{r^{2}}+\frac{3 \hbar^{2}}{16 m} \frac{1}{r^{3}} \quad r \rightarrow \infty,
$$


or

$$
V_{P \infty}=\frac{e^{2}}{r}-\frac{3 \hbar^{2}}{32 m} \frac{1}{r^{2}} \quad r \rightarrow \infty .
$$

Having demonstrated this DFT result for the leading term in the $1 / Z$ expansion for spherical atomic ions, we shall explore below a corresponding form to Eq. (6) for closed shells generated by three-dimensional harmonic confinement, which is also analytically tractable. It is noteworthy that magnetically trapped dilute ultracold fermion vapors are now studied experimentally starting with the pioneering work of DeMarco and Jin [9]. This allows, in fact, harmonically confined fermions to be studied as a function of dimensionality $d \leqslant 3$. The theoretical work of Howard et al. [10] has been motivated by the above experimental progress. In Ref. [10] and in $d$ dimensions, the functional derivative of the single-particle kinetic energy $T_{s}[n]$ of DFT is obtained in their Eq. (19) as

$$
\frac{\delta T_{s}}{\delta n(r)}=\frac{n^{2 / d}(r)}{4 d} \frac{\hbar^{2}}{m} \int^{r} \frac{1}{n^{1+2 / d}(s)} \frac{\partial \nabla^{2} n(s)}{\partial s} d s .
$$

The Pauli potential $V_{P}$ can then be obtained, as mentioned already in the Abstract, as

$$
V_{P}(r)=\frac{\delta T_{s}}{\delta n(r)}-\frac{\delta T_{W}}{\delta n(r)} .
$$

Since $T_{W}=\int t_{W}(r) d \mathbf{r}$, we know the last term in Eq. (12) explicitly. Also, from Eq. (10) of [10], but now specialized to $d=3$,

$$
\frac{\partial t_{G}}{\partial r}=-\frac{3}{2} n \frac{\partial V}{\partial r} .
$$

Returning to Eq. (1) above, we can use this $3 D$ result (13) for harmonic confinement to remove the term $n \frac{\partial V}{\partial r}$ to yield

$$
\frac{2}{3} \frac{\partial t_{G}}{\partial r}+\frac{\hbar^{2}}{4 m} \frac{\partial \nabla^{2} n}{\partial r}=4 \frac{t_{W}(r)}{r}+2 \frac{\partial t_{W}(r)}{\partial r}+n(r) \frac{\partial V_{P}}{\partial r} .
$$

The resemblance of Eq. (14) to Eq. (6) is remarkable though they are not identical.

In conclusion, it is stated that for the leading Coulombic term in the $1 / Z$ expansion for spherical atomic ions, the Pauli potential is written in terms of the kinetic energy density, the electron density, and its low-order derivatives. It is also demonstrated that the equation derived for the Pauli potential of an arbitrary number of closed shells with purely harmonic confinement has a very similar form.

\section{ACKNOWLEDGMENTS}

Á.N. acknowledges Grant OTKA No. K 67923.
[1] N. H. March and A. Nagy, Phys. Rev. A 78, 044501 (2008).

[2] M. Cohen, Adv. At. Mol. Phys. 25, 195 (1988).

[3] N. H. March, Phys. Rev. A 33, 88 (1986).

[4] T. Kato, Commun. Pure Appl. Math. 10, 151 (1957).

[5] C. Amovilli and N. H. March, Phy. Chem. Liquids 38, 505 (2000).
[6] R. G. Parr and W. Yang, Density Functional Theory of Atoms and Molecules (Oxford University Press, New York, 1989).

[7] O. J. Heilmann and E. H. Lieb, Phys. Rev. A 52, 3628 (1995).

[8] N. H. March, Phys. Rev. A 54, 5415 (1996).

[9] B. DeMarco and D. S. Jin, Science 285, 1703 (1999).

[10] I. A. Howard, N. H. March, and L. M. Nieto, Phys. Rev. A 66, 054501 (2002) 